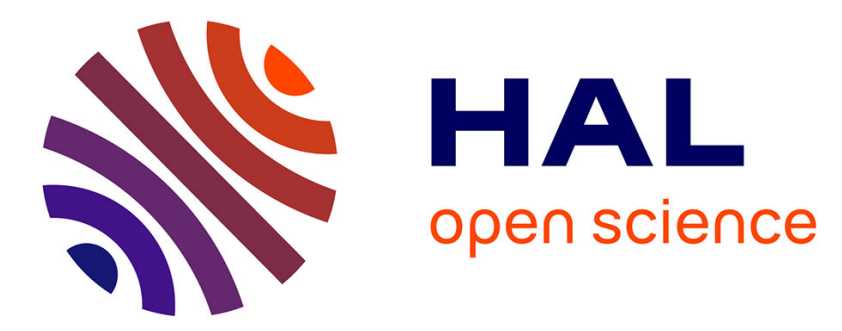

\title{
Effect of the Acoustic Impedance Mismatch at the Bone-Soft Tissue Interface as a Function of Frequency in Transcranial Ultrasound: A Simulation and In-Vitro Experimental Study
}

Shreyank Gupta, Guillame Haiat, Catherine Laporte, Pierre Belanger

\section{To cite this version:}

Shreyank Gupta, Guillame Haiat, Catherine Laporte, Pierre Belanger. Effect of the Acoustic Impedance Mismatch at the Bone-Soft Tissue Interface as a Function of Frequency in Transcranial Ultrasound: A Simulation and In-Vitro Experimental Study. IEEE Transactions on Ultrasonics, Ferroelectrics and Frequency Control, inPress. hal-03084611

\section{HAL Id: hal-03084611 \\ https://hal.science/hal-03084611}

Submitted on 21 Dec 2020

HAL is a multi-disciplinary open access archive for the deposit and dissemination of scientific research documents, whether they are published or not. The documents may come from teaching and research institutions in France or abroad, or from public or private research centers.
L'archive ouverte pluridisciplinaire HAL, est destinée au dépôt et à la diffusion de documents scientifiques de niveau recherche, publiés ou non, émanant des établissements d'enseignement et de recherche français ou étrangers, des laboratoires publics ou privés. 


\title{
Effect of the Acoustic Impedance Mismatch at the Bone-Soft Tissue Interface as a Function of Frequency in Transcranial Ultrasound: A Simulation and In-Vitro Experimental Study
}

\author{
Shreyank Gupta ${ }^{1,2}$, Guillame Haiat ${ }^{1,2}$, Catherine Laporte $^{1}$, Pierre Belanger ${ }^{1}$ \\ École de technologie supérieure, Montreal, Canada ${ }^{1}$ \\ Email: shreyank.gupta.1@ens.etsmtl.ca \\ MSME, CNRS UMR 8208, Univ Paris Est Creteil, Univ Gustave Eiffel, F-94010 Creteil, France ${ }^{2}$
}

\begin{abstract}
Transcranial Doppler ultrasound (TCD) is a method that uses a hand held low frequency $(2-2.5 \mathrm{MHz})$, pulsed Doppler phased array probe to measure blood velocity within the arteries located inside the brain. The problem with TCD lies in the low ultrasonic energy penetrating inside the brain through the skull which leads to low signal to noise ratio. This is due to several effects including phase aberration, variations in the speed of sound in the skull, scattering, the acoustic impedance mismatch and absorption of the three layer medium constituted by soft tissues, the skull and the brain. The goal of this paper is to study the effect of transmission losses due to the acoustic impedance mismatch on the transmitted energies as a function of frequency. To do so, wave propagation was modelled from the ultrasonic transducer into the brain. This model calculates transmission coefficients inside the brain, leading to a frequency-dependent transmission coefficient for a given skin and bone thickness. This approach was validated experimentally by comparing the analytical results with measurements obtained from a bone phantom plate mimicking the skull. The average position error of the occurrence of the maximum amplitude between the experiment and analytical result was equivalent to a $0.06 \mathrm{~mm}$ error on the skin thickness given a fixed bone thickness. Similarity between the experimental and analytical result was also demonstrated by calculating correlation coefficients. The average correlation between the experimental and analytical result came out to be 0.50 for a high frequency probe and 0.78 for a lower frequency probe. Further analysis of the simulation showed that an optimized excitation frequency can be chosen based on skin and bone thicknesses, thereby offering an opportunity to improve the image quality of TCD.
\end{abstract}

\section{INTRODUCTION}

Stroke is the second leading cause of death in developed nations, and, in Canada, one person dies every 7 minutes from stroke [7]. There are other diseases like the sickle disease, vasospasm, etc., which further adds to the statistics of the death rate caused by cerebrovascular disease. There is, therefore, a need for a system which can be used often to perform diagnosis and monitoring which provides quick, reliable information about a patient's health. Transcranial Doppler (TCD) ultrasound (US) imaging is a promising, non-invasive technique which helps in diagnosing cerebrovascular diseases of the brain by detecting variations in the blood velocity and is usually done using a standard cardiac probe whose centre frequencies range from 2-2.5 $\mathrm{MHz}$ [2]. The blood velocity is measured by the reflections of the moving red blood cells through the Doppler effect. One of the limitations of TCD is that diseases like vasospasm and stroke require accurate measurement of the blood flow, therefore requiring the diameter of the vessel to be measured. If TCD can detect that there is blockage in the Middle cereberal artery (MCA) by determining the flow, then it is highly probable that an acute stroke occur within 5 hours. Adding to that, less than $10 \%$ of stroke patients are treated accurately because the current TCD systems have poor image quality which makes blood flow measurement difficult for early detection [3].

Poor ultrasound transmission in TCD is mostly due to the presence of the skull in the wave propagation path, which leads to several adverse effects. Those effects include phase aberration, variations in the speed of sound in the skull, scattering, the acoustic impedance mismatch and absorption. Two effects are considered in this study: (1) attenuation and (2) acoustic impedance mismatch. The acoustic impedance mismatch has a complex relationship with the frequency whereby there is a loss in the transmitted energy at the different interfaces along the wave propagation path. The effect is shown in Fig. 1, which was obtained via an analytical model described in section II-A and shows how much acoustic energy is transmitted through skin and bone at $2 \mathrm{MHz}$. For many combinations of skin and bone thickness, the model predicts near-zero energy transmission, which is due to the acoustic impedance mismatch between the skin and the bone. Image formation would not be feasible with such low energies. There is also a strong loss of signal because the attenuation in the skull is high and is a function of frequency. Furthermore, due to attenuation caused by the skull bone, the amplitude of the received echoes reduces rapidly as the depth in the brain increases. This causes a low signal to noise ratio (SNR) which directly correlates to the contrast in the images. Because of this low contrast, clinicians are not always able to see the vessels in images, making blood 
flow measurement difficult. Clinical studies demonstrated that TCD was successful in only $38.1 \%$ of patients [5].

In the past, much work was done to improve the energy transmitted through the skull, mainly considering the attenuation effect [21]-[23], and time reversal techniques [31]-[35] but the acoustic impedance mismatch effect was typically ignored. Yue et. al [20] designed a probe for TCD that improves the transducer sensitivity and the bandwidth but used the typical TCD frequency range. This probe improve the image resolution because it works on a wide range of frequencies, but the problem of poor transmission through the temporal bone still persists since neither attenuation nor acoustic impedance mismatch were taken into account. Klotzsch et.al [6] studied how to improve transcranial imaging and claimed that a 1 $\mathrm{MHz}$ probe allows a better insonation of patients. Their study showed that vessels can be characterized in only $25 \%$ of patients using a $2 \mathrm{MHz}$ probe but success rate was close to $100 \%$ when using a 1-MHz probe. This study showed that adapting the frequency in TCD may improve the success rate. Since the transmitting frequency was reduced to $1 \mathrm{MHz}$, the resolution of the images worsened. Lindsey et. al [21], [22] designed custom 2D sparse array transducers. They placed the transducer arrays on either side of the temporal acoustic windows and combined the received signals from both sides to make one final image. By doing so, they eliminated the effect of asymmetry on either side of the window. Aarnio et. al [11] showed the occurrence of spectral peaks in transmission in real skulls in five out of six anatomical sites. However the effect of an acoustic impedance mismatch at the bone-soft tissue interface was ignored in the selection of the frequency. Optimization of the frequency based on the acoustic impedance mismatch is therefore still an open question. White et. al [10], Aarnio et. al [11] and, Clement et. al [12] used analytical models to study phase shift and attenuation through the skull. These studies were performed on different locations of the skull, and depicted the variation of ultrasound energy as a function of skull thickness and phase shifts. However, these studies neglected the effect of the skin thickness on the acoustic impedance mismatch. Hynynen et. al [23] did a study on excitation frequency selection for transcranial imaging. They specifically mentioned aiming for frequencies below $1 \mathrm{MHz}$. There were two reasons behind this: (1) the scattering of the US, because parameters like density and bulk modulus of blood differ greatly from that of it's surrounding tissues [8], [9], and (2) the attenuation caused by the skull [4]. Scattering and attenuation cause loss in the energy of the signal. They also considered the use of shear waves to enhance US transmission through the skull, as this improves acoustic impedance matching, and reduces refraction and phase alteration compared to longitudinal waves [23]. But the problem with this method is that the amplitude of shear waves is lower than that of longitudinal waves, which results in poor SNR, and low contrast in the images. Apart from these effects, researchers in recent years have also aimed for correcting ultrasound waveform aberration caused by the skull [30]. A technique that has attracted the attention of many researchers is the time reversal technique [31]-[35]. The technique computes the delays for the elements of the probe from MRI and CT mapping of the skull. This technique improves the contrast and spatial resolution of the images, but since each person has different skull properties, it is impractical to use in an intensive care setting.

The effect of the frequency dependent acoustic impedance mismatch at the bone-soft tissue interface has been typically ignored in the discussed literature. Therefore, the aim of this paper is to isolate the frequency-dependent effect of acoustic impedance mismatch in a relatively simple situation which in turn relates to optimizing the transmitted frequency for TCD. Once the optimal frequency is chosen with respect to the acoustic impedance, the effect of other spatially varying acoustical properties will indeed be important. They will however be significant at all frequencies. Therefore, the choice of the acoustic impedance dependent optimal frequency will always be beneficial. The present study is an important step towards understanding the reasons behind the poor ultrasound transmission in TCD and furthermore devise a methodology to improve TCD imaging.

\section{MATERials AND Methods}

\section{A. Analytical model}

An analytical model [28] for which a schematic description is presented in Fig. 2 was used to simulate the acoustic energy transmission as a function of the frequency in a multi-layer transcranial acoustic wave propagation transmission model. This paper is focused on plane wave transmission to eventually use plane wave Doppler imaging [13]. Though most clinical systems use focused ultrasound for Doppler measurements, plane wave TCD imaging is proposed due to its capability to acquire fast velocity information without aliasing. Clinical systems such as the Aixplorer [14] are now starting to offer plane wave imaging due to its superior frame rate. Therefore, a longitudinal plane wave is generated from a piezoelectric element, traveling from left to right through a series of layers with different acoustic impedances $Z$. The plane wave interacts with layers causing multiple reflections and transmissions until a transmitted wave reaches the brain. The brain and piezoelectric crystal layers are assumed to have a semi-infinite thickness. The acoustic impedances of the piezoelectric element, the brain, the bone, the skin, and the matching layer are $Z_{p}$ (34.20 MRayl), $Z_{m}$ (7.10 MRayl), $Z_{s}$ (1.50 MRayl), $Z_{b}$ (7.00 MRayl), and $Z_{\text {brain }}(1.50 \mathrm{MRayl})$ respectively [25]. Since the brain consists mainly of water, an acoustic impedance of 1.50 MRayl was assumed. The skin's acoustic properties also matches very closely with water [18]. While, the absorption of skin due to scalp tissue [15] is significantly different from that of water, the effect of absorption would remain almost constant for the given small range of frequencies typically identified as optimal as shown in Fig. 1 in which the frequency used was $2 \mathrm{MHz}$. The acoustic impedance in skin was shown to vary within a narrow range [18]. However, the acoustic impedance of the human skull was shown to vary significantly [4]. Although the bone acoustic impedance was constant at 7 MRayl in this paper, the concept of adapting the centre frequency of the transmitted signal to acoustic impedances of the layers along the wave propagation path is of interest. The thicknesses of the matching layer, the skin, and the bone are $t_{m}, t_{s}$, and $t_{b}$ 


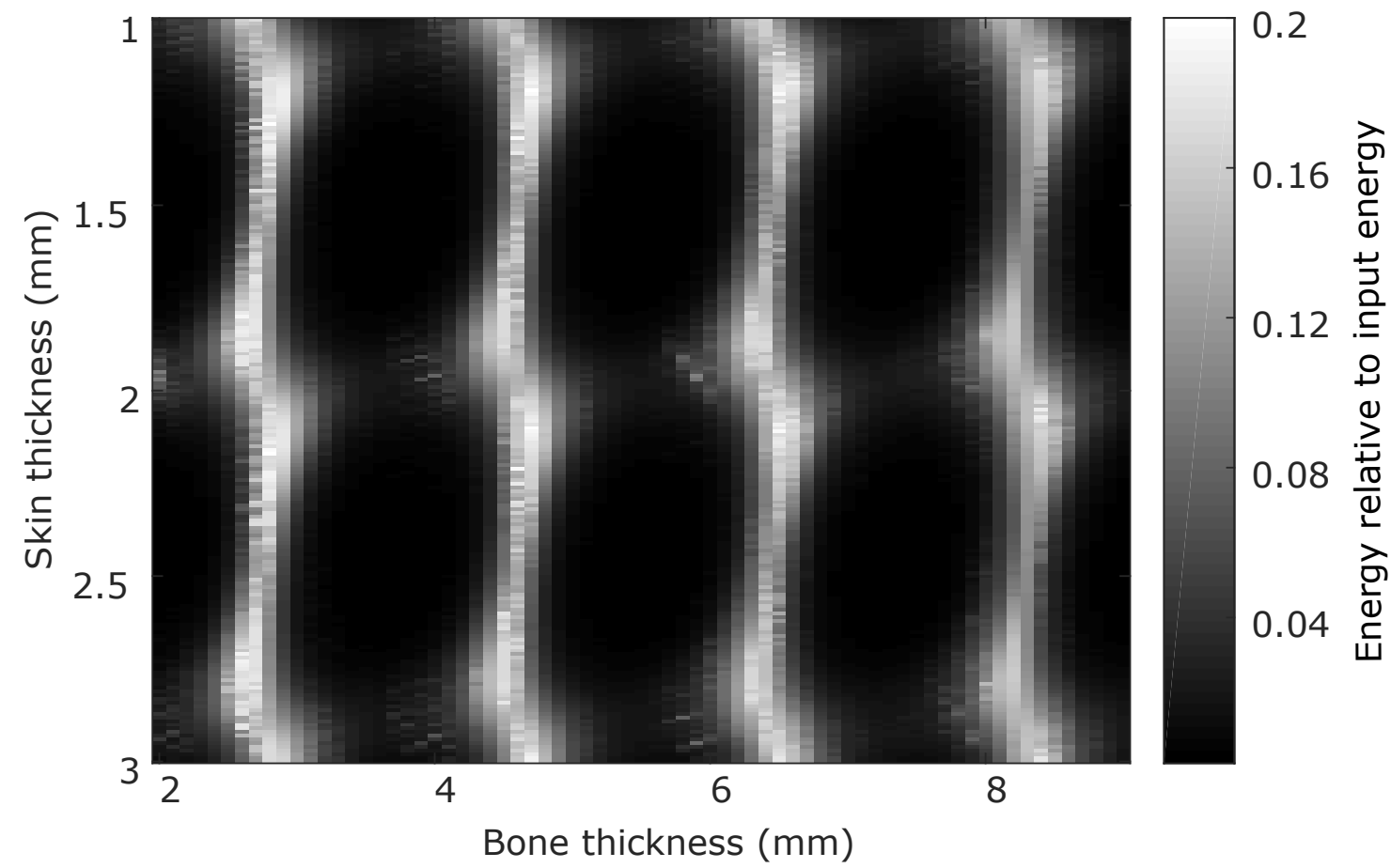

Fig. 1: Transmitted energy as a function of skin and bone thickness. The figure shows a simulated map of the ultrasound energy transmitted to the brain using a centre frequency of $2 \mathrm{MHz}$.

respectively. The matching layer thickness was considered to be $\lambda / 4$ at a centre frequency of $3 \mathrm{MHz}$ for a $2-4 \mathrm{MHz}$ probe and at $1.50 \mathrm{MHz}$ for a 1-2 MHz probe. However, the thickness of skin and bone do vary in the literature [17], [19], which motivates the study presented in this paper. The assumed speed of sound in the piezoelectric element layer, the matching layer, the skin layer and the bone layer is $4500 \mathrm{~m} / \mathrm{s}, 3310 \mathrm{~m} / \mathrm{s}$ for a 2-4 MHz probe and $2000 \mathrm{~m} / \mathrm{s}$ for $1-2 \mathrm{MHz}$ probe (provided by the manufacturer), $1500 \mathrm{~m} / \mathrm{s}$ and $3000 \mathrm{~m} / \mathrm{s}$ (provided by the manufacturer) respectively. In order to predict the energy inside the brain based on different thicknesses and acoustic impedances, the classical transmission matrix method was used [28]. If a layer has a thickness $t_{l}$ and acoustic impedance $Z_{l}$, the transmission matrix is given by

$$
\left[T_{l}\right]=\left[\begin{array}{cc}
\cos \theta_{l} & i Z_{l} \sin \theta_{l} \\
i \sin \theta_{l} / Z_{l} & \cos \theta_{l}
\end{array}\right],
$$

where $\theta_{l}=2 \pi t_{l} / \lambda_{l}, \lambda_{l}$ is the wavelength and $t_{l}$ is the thickness of the $l_{t h}$ layer. The equivalent acoustic impedance of the model is $\left(Z_{e q}\right)$ which considers the acoustic impedance of piezoelectric element:

$$
Z_{e q}=\frac{T_{11} \cdot Z_{p}+T_{12}}{T_{21} \cdot Z_{p}+T_{22}}
$$

where $T_{m n}, m, n \in\{1,2\}$ are the elements of a transmission matrix $T_{x}$ representing a multi-layered structure consisting of a matching layer, a skin layer and a bone layer. $T_{x}$ is given by:

$$
\left[T_{x}\right]=T_{1} \cdot T_{2} \cdot T_{3}=\left[\begin{array}{ll}
T_{11} & T_{12} \\
T_{21} & T_{22}
\end{array}\right]
$$

where 1,2,3 represents the matching, the skin and the bone layers. For a given TCD wave propagation model, transmission coefficients are calculated. The coefficients of the reflected and transmitted waves in the brain are given by:

$$
R=\left[\frac{\left|Z_{e q}-Z_{\text {brain }}\right|}{\left|Z_{e q}+Z_{\text {brain }}\right|}\right]^{2}
$$

and

$$
T=1-R
$$

For maximum energy transmission, $\mathrm{R}=0$, and $\mathrm{T}=1$; i.e., $Z_{e q}=Z_{\text {brain }}$ in equation 2 . In the case of TCD, this situation can happen when the thickness of the matching layer $\lambda_{m} / 4$ is followed by skin and bone thicknesses of $t_{s}=\lambda_{s} / 2$ and $t_{b}=\lambda_{b} / 2$ respectively. $\left(\lambda_{m}, \lambda_{s}, \lambda_{b}\right.$ are the wavelengths in the matching, the skin and the bone layers respectively). Thus, certain combinations of frequency, skin thickness, bone thickness and matching layer thickness lead to better energy transmission than others. The most obvious parameter to tune in a practical situation where different patients are seen would be the frequency. Therefore, the analytical simulation was run using equations $1,2,3$ which provided the transmission coefficients for different bone, skin and matching thicknesses as a function of frequency. The energy propagating back to 
Transcranial acoustic wave propagation model

\begin{tabular}{|l|l|l|l|l|}
\hline \multicolumn{2}{|c|}{ Probe } & Ultrasonic wave propagation path \\
\begin{tabular}{|l|l|l|} 
Piezoelectric \\
element
\end{tabular} & Matching layer & Skin & Bone & Brain \\
$Z_{\mathrm{P}}=34.20 \mathrm{MRayl}$ & $\mathrm{Z}_{\mathrm{m}}=\sqrt{\mathrm{Z}_{\mathrm{p}} \times \mathrm{Z}_{\text {brain }}}$ & $\mathrm{Z}_{\mathrm{s}} \approx 1.50 \mathrm{MRayl}$ & $\mathrm{Z}_{\mathrm{b}} \approx 7.00 \mathrm{MRayl}$ & $\mathrm{Z}_{\text {brain }} \approx 1.50 \mathrm{MRayl}$ \\
& & & & \\
\hline
\end{tabular}

Fig. 2: A one-dimensional acoustic wave propagation model for TCD. The acoustic impedance of the piezoelectric element, the matching layer, the skin layer, the bone layer and the brain are denoted by $Z_{p}, Z_{m}, Z_{s}, Z_{b}$, and $Z_{\text {brain }}$. The thickness of the matching layer, the skin layer and the bone are denoted by $t_{m}, t_{s}$ and $t_{b}$

the probe from a reflector is of interest and is also calculated using equations $1,2,3$.

The transmission coefficients received from the analytical model were based on the assumption that the input excitation signal is a sine wave of a single frequency leading to an infinite signal in the time domain. In practice, the signals are finite in the time domain. To overcome this effect:

1) A transmitted energy coefficients curve is calculated for different skin thicknesses as a function of frequency based on the analytical model.

2) To find the transmitted energy of a finite time domain signal, a 10-cycle Tukey-windowed tone burst sampled at $10 \mathrm{MHz}$ is simulated as an excitation signal. The power spectrum of the input signal and transmission coefficient spectrum are multiplied to provide the transmitted energy spectrum;

3) The transmitted energy spectrum is multiplied by the attenuation curve which is proportional to the frequency squared and is derived using $(30 \mathrm{~dB} / \mathrm{cm} @ 2.25 \mathrm{MHz}$ [24] in the case of the bone phantom plate used in the experiments. The attenuation at $2.25 \mathrm{MHz}$ was supplied by the manufacturer of the phantom). It was assumed that the attenuation was constant in the skull layer and the value of attenuation was extrapolated for different centre frequencies ranging from 1-4 MHz. The absorption was not considered in the modeling since it would unlikely change the optimal frequency with respect to the acoustic impedance mismatch due to skin thickness. Across the small range of optimal frequencies obtained from the model, the absorption would be almost constant.

4) Reflected energy is calculated all the way back to the piezoelectric element from an aluminium plate with a reflection coefficient of $86 \%$ [25].

The final spectrum gives the reflected energy with respect to the input signal which includes the combined effect of attenuation and the acoustic impedance mismatch. This step is repeated for different skin thicknesses varying from 1 to $2 \mathrm{~mm}$ for a constant bone thickness of $4.40 \mathrm{~mm}$ giving a 2D map as shown in Fig. 3 (a) and (b) as a function of the centre frequency ( $1-3 \mathrm{MHz}$ ). The experimental validation was limited to a constant bone thickness of $4.40 \mathrm{~mm}$ which is also in the range of the average human temporal bone thickness [19], with centre frequencies ranging from $1-2 \mathrm{MHz}$ and 2$3 \mathrm{MHz}$. The two sets of frequencies were chosen to perform simulations and experiments. Lower frequencies were chosen as it was suggested in the literature that TCD imaging performs better in lower frequency ranges. The second set was chosen because the typical frequency range used for TCD is between 2-2.5 MHz. The skin thickness was kept between 1 to $2 \mathrm{~mm}$ since the average skin thickness of a human being near the temporal window is $1.85 \mathrm{~mm}$ [17]. Fig. 3 (a) and (b) were used for the comparison in the next sections to compare analytical and experimental results.

\section{B. Experiment}

To validate the analytical model, an experimental setup was devised. Fig. 4 shows the different components which were used to make the setup. A corner bracket was used to hold a linear positioning stage (Velmex A1503P20-S1.5, $0.025 \mathrm{~mm}$ resolution) vertically. The probe holder was fixed to the adapter using set screws. The Verasonics $\mathrm{P} 4-2 \mathrm{v}$ probe was connected to the Verasonics V1 phased array controller and could be moved vertically using the linear stage. The whole assembly was immersed in a water tank, which also contained a bone phantom plate [24] made up of a ceramic material mimicking the acoustic properties $\mathrm{v}_{l}: 3000 \mathrm{~m} / \mathrm{s}, \rho: 2100 \mathrm{~kg} / \mathrm{m}^{3}$ - the cortical layer of the bone phantom can be assumed homogeneous. However, the trabecular bone layer contains voids and inclusions to increase its attenuation. The total attenuation of the bone phantom plate is $30 \mathrm{~dB} / \mathrm{cm}$ @ $2.25 \mathrm{MHz}$ of the skull. The plate thickness was $4.40 \mathrm{~mm}$. An aluminium block was chosen as a reflector at a distance of $3 \mathrm{~cm}$ from the bone surface. The tank was filled with water to mimic the skin's 

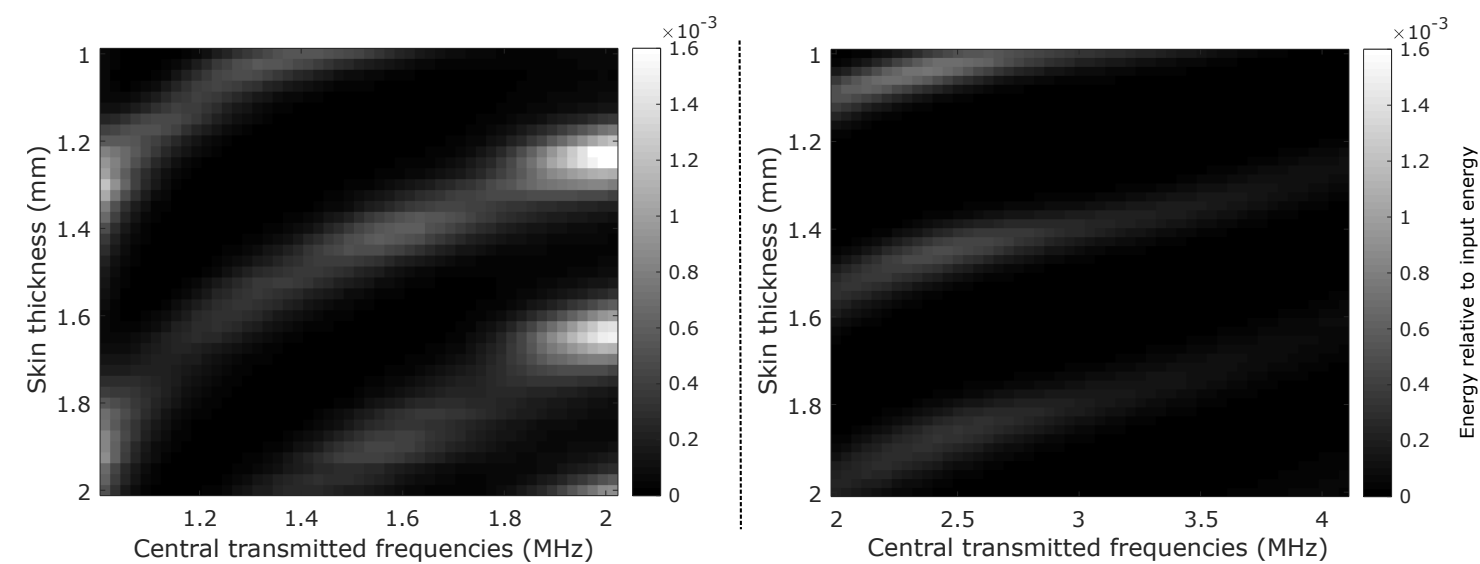

Fig. 3: (a) Received energy as a function of skin thickness varying from 1 to $2 \mathrm{~mm}$ and central transmitted frequencies varying from 1 to $2 \mathrm{MHz}$. The reflection is caused by an aluminium plate $3 \mathrm{~cm}$ from the bone surface. (b) Received energy as a function of skin thickness varying from 1 to $2 \mathrm{~mm}$ and frequencies from 2 to $3 \mathrm{MHz}$. Both 2D maps were obtained from simulations.

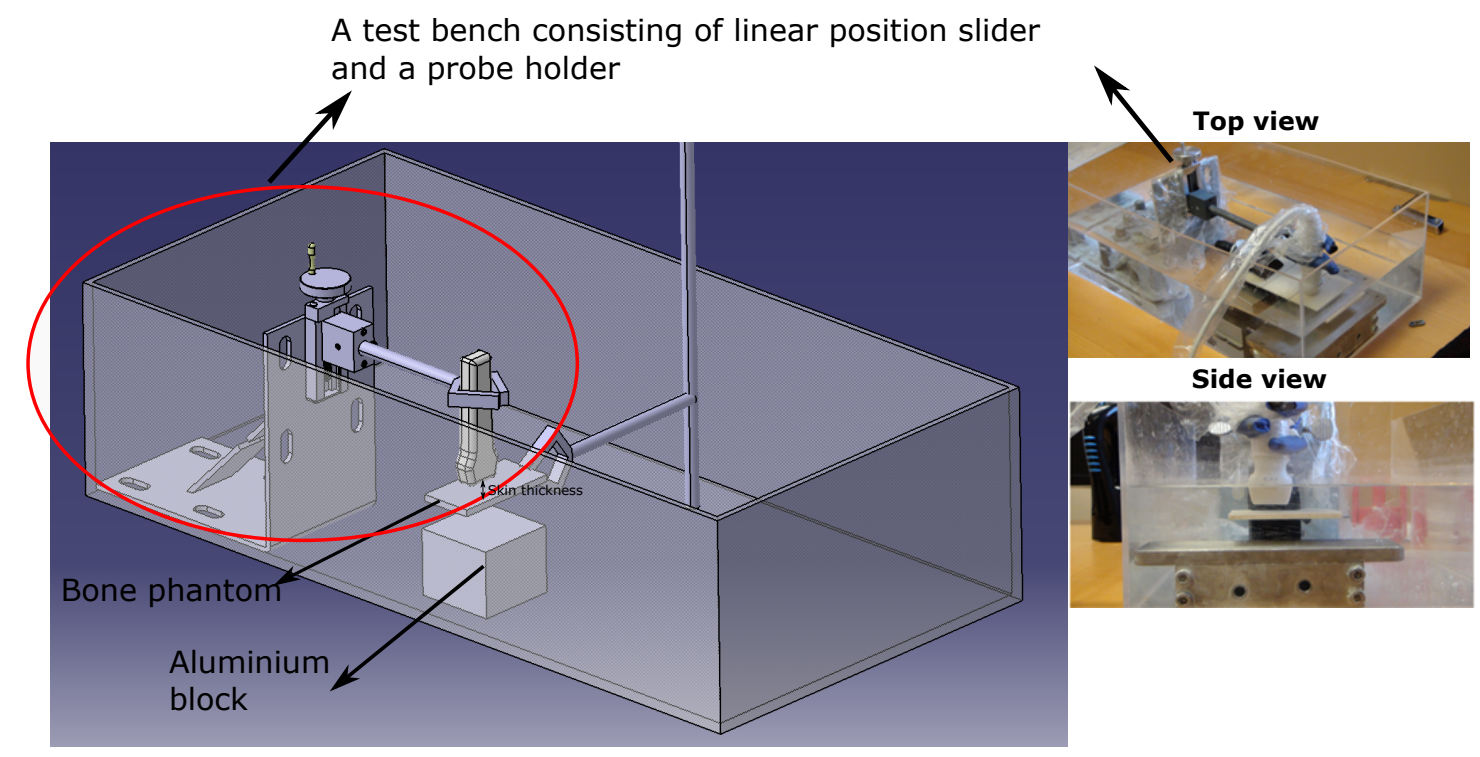

Fig. 4: The experimental setup comprised of a linear positioning stage to hold a US probe, a bone phantom plate which mimicked the acoustic properties of the skull, water which mimicked the skin's acoustic properties and an aluminium block which was used as a reflector.

acoustic impedance. The experiments were conducted using a Verasonics V1 system [16]. The frequency range of the P4-2v probe was $2-4 \mathrm{MHz}$ with the centre frequency at $3 \mathrm{MHz}$. This probe had 64 elements which were simultaneously activated to approximate a plane wave. The experimental procedure was repeated for the 1.5L64-0.3x15 probe manufactured by Guangzhou Doppler Electronic Technologies Inc.. This second probe had a frequency bandwidth between 1 and $2 \mathrm{MHz}$. In all cases, the transmitted waveform was a 10-cycle Tukey windowed tone burst. The initial position of the probe was marked at the top of the bone phantom plate and then it was moved up step by step, thus increasing the gap between the probe and bone phantom plate. This gap mimics the skin thickness. A typical transmitted waveform without the bone phantom is shown in fig. 5 (a) A typical received waveform for a skin thickness of $1 \mathrm{~mm}$ is shown in Fig. 5 (b). The probe was moved up in steps of $0.025 \mathrm{~mm}$ to acquire a measurement. The probe was moved upwards until the gap between the probe and the plate was $2 \mathrm{~mm}$. In Fig. 5 (b), the received waveform acquired using the $\mathrm{P} 4-2 \mathrm{v}$ probe contains a reflection from the top part of the bone plate and from the surface of the aluminium block. The reflected signal from the aluminium block is shown in the small box of Fig. 5 (b). The 10 cycles of the reflected signal were extracted. The input signal is the emitted signal from the phased array controller. The ratio of the reflected to input signal energy was calculated using equation 


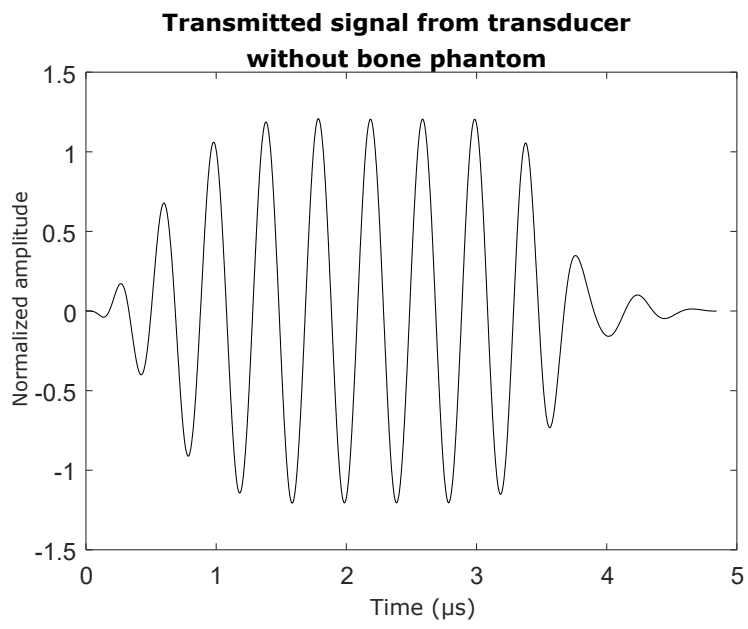

(a)

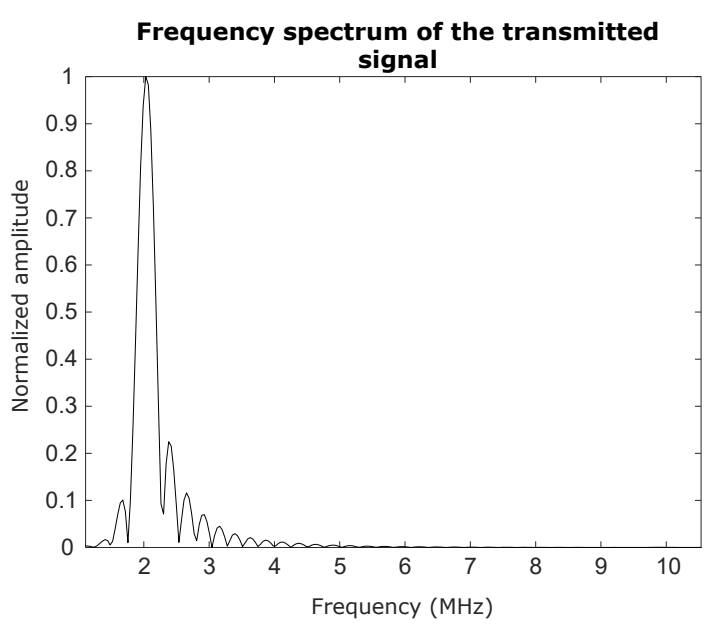

(b)

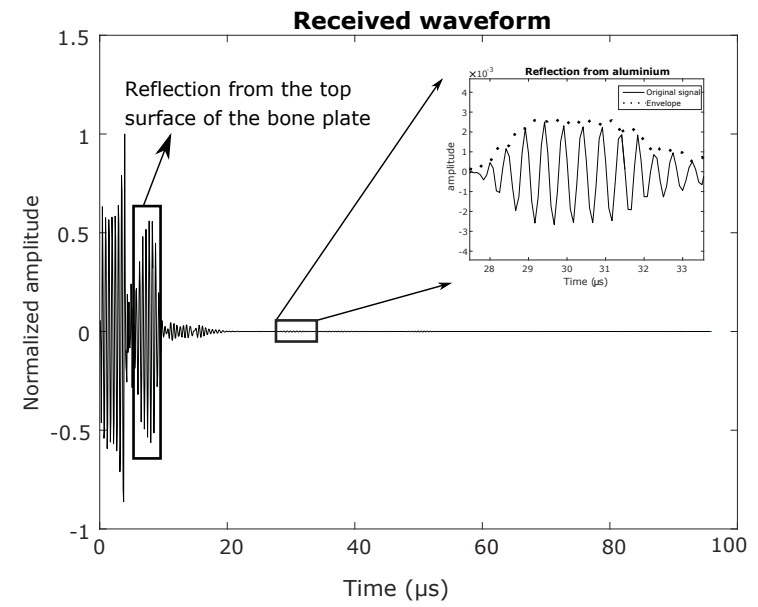

(c)

Fig. 5: (a) A typical transmitted waveform for a frequency of $2 \mathrm{MHz}$ without the presence of bone phantom. (b) Corresponding frequency spectrum of the transmitted waveform without bone phantom. (c) A time trace of a received waveform for a frequency of $2 \mathrm{MHz}$ and a gap between the probe and the bone phantom plate of $1 \mathrm{~mm}$. The figure also shows a reflection from the aluminium block along with the envelope.

4

$$
E_{\text {brain }}=\left|\frac{\mid \mathrm{FFT}\left(\text { signal }_{\text {extracted }}\right) \mid}{\mid \mathrm{FFT}(\text { input }) \mid}\right|^{2} .
$$

This same calculation was repeated for the other input signal centre frequencies ranging from 2-3 MHz for the P4-2v probe and from 1 to $2 \mathrm{MHz}$ for the $1.5 \mathrm{~L} 64-0.3 \times 15$ probe. The variation of received energy as a function of the frequency for a skin thickness of $1 \mathrm{~mm}$ is shown in Fig. 6. The same process was repeated for multiple skin thicknesses varying from 1-2 mm keeping the bone thickness constant. This experiment provides a two-dimensional map, as shown in Fig. 7. The experimental setup and measurement process mimic the analytical model results with a constant bone thickness and skin thickness varying from 1 to $2 \mathrm{~mm}$.

\section{RESULTS}

To look more closely at the comparison between the analytical and experimental results, narrow band signals with a centre frequency between $1.00 \mathrm{MHz}$ to $1.19 \mathrm{MHz}$ and $2.06 \mathrm{MHz}$ to $2.26 \mathrm{MHz}$ at regular intervals of $65 \mathrm{kHz}$ were taken from the original data and overlaid on each other as shown in Fig. 8. Table I shows the correlation coefficient between the simulated and experiment results for the given frequencies, which was calculated according to

$$
\frac{1}{n-1}\left(\sum_{x} \sum_{y} \frac{(x-\bar{x})(y-\bar{y})}{S_{x} S_{y}}\right),
$$

where $x$ and $y$ are the two signals, $\bar{x}$ and $\bar{y}$ are the mean of the two vectors , $S_{x}$ and $S_{y}$ are the standard deviation of the two vectors and $n$ is the length of the signals. 


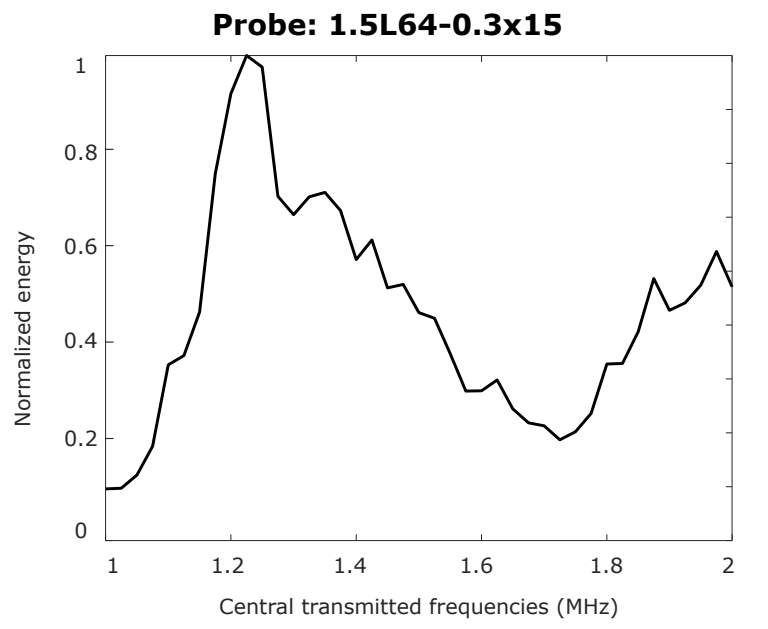

(a)

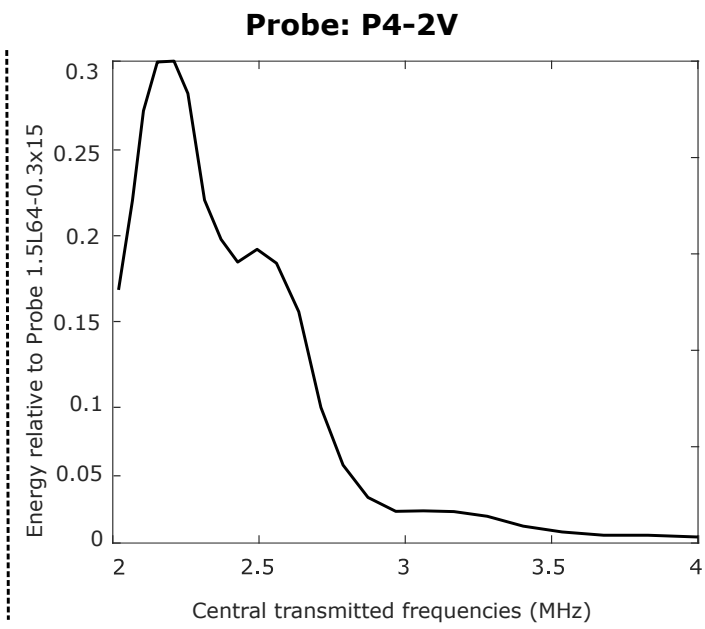

(b)

Fig. 6: (a) Normalized experimental energy received as a function of the frequency for a $1 \mathrm{~mm}$ skin thickness for probe 1.5L64$0.3 \times 15$. (b) Experimental energy received as a function of the frequency for a $1 \mathrm{~mm}$ skin thickness for probe P4-2v relative to probe $1.5 \mathrm{~L} 64-0.3 \times 15$.
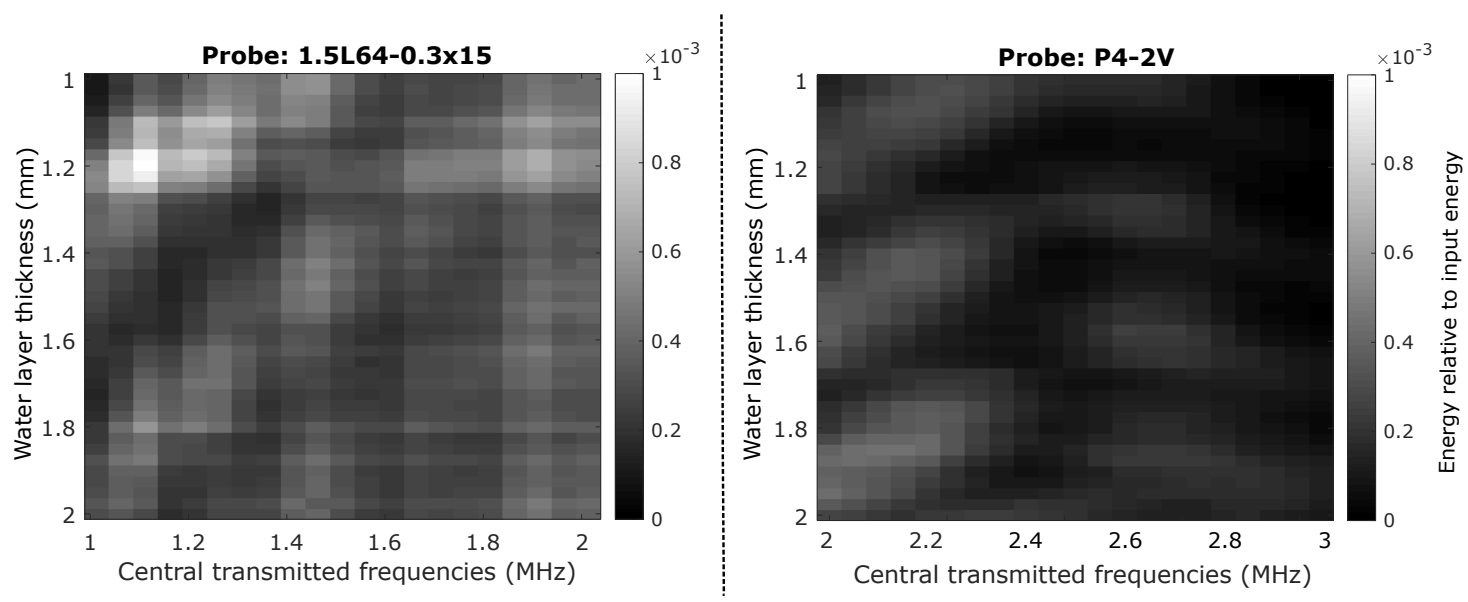

Fig. 7: The experimental 2-D map shows the variation of received energies as a function of frequency and skin thickness. The experimental result showed that there is an acoustic impedance mismatch effect as the intensities vary as a function of skin thickness for a constant frequency.

The table also contains the average local shift (the average skin thickness difference) between simulation and experiment of the received energy maxima and its standard deviation. The difference between the position of the maximum amplitude between the experiment and analytical result was equivalent to an average of $0.06 \mathrm{~mm}$ error on the skin thickness given a fixed bone thickness. The average correlation between the experimental and analytical result came out to be 0.50 for a high frequency probe and 0.78 for a lower frequency probe. The Average full-width at half maximum (FWHM) for experimental and analytical curves was calculated to show the extent of the spread of energy around the maximum. The average statistic was used because it summarized all the individual local shifts, standard deviation and FWHM into a single value, which made it easy to compare between all the curves. These results showed that the energies do depend on specific combinations of bone and skin thicknesses as a function of the frequency.

\section{Discussion}

The aim of this paper was to show the effects of the frequency-dependent acoustic impedance mismatch. By choosing the frequency that will enable the highest transmission of ultrasonic energy the chances of a successful measurement are higher. While there are effects like scattering, shear mode 

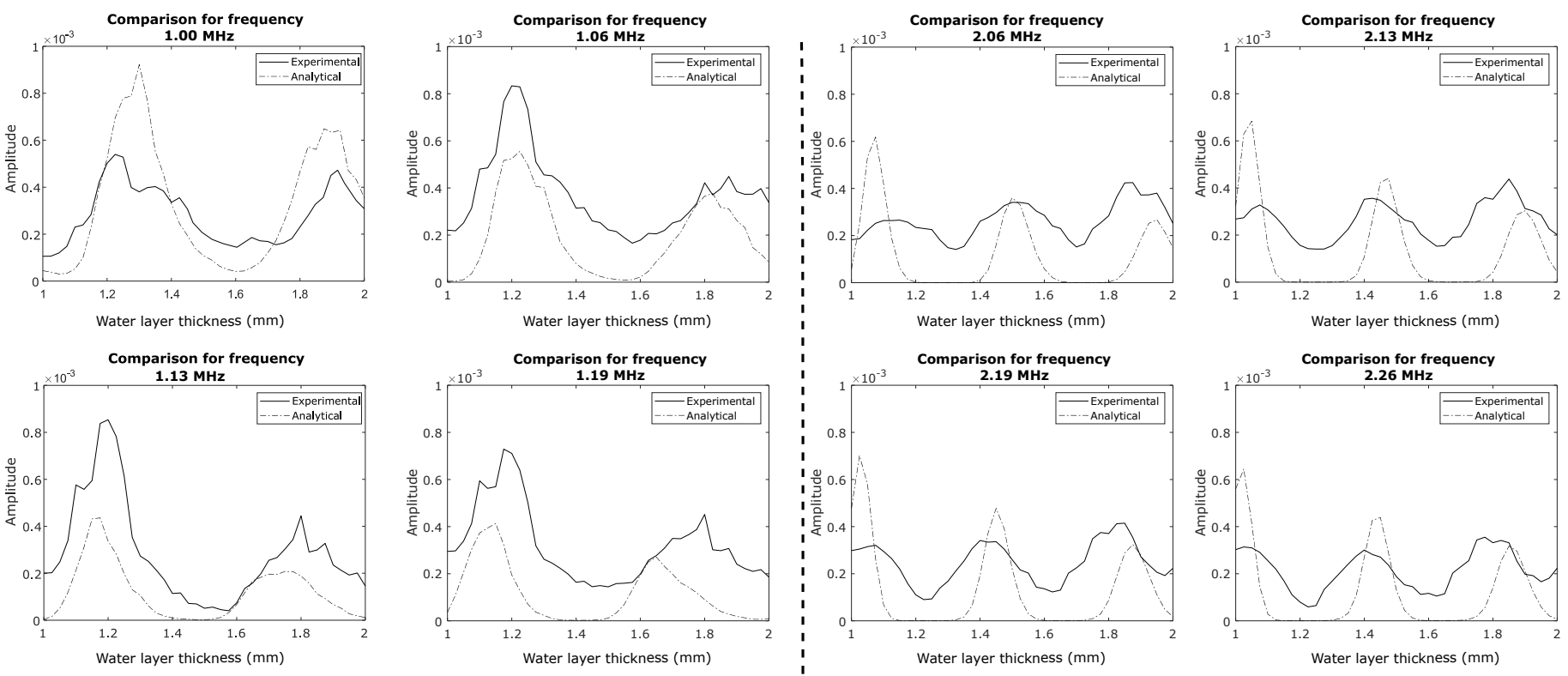

(A)

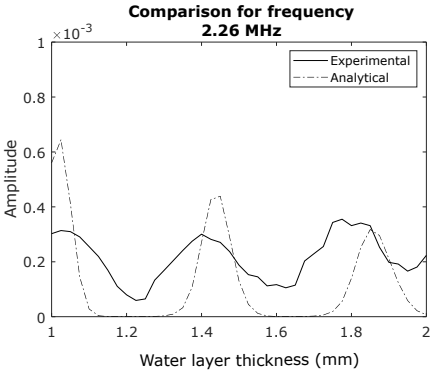

(B)

Fig. 8: The multiple figures shows the comparisons between the selected frequencies ((A) being the results from probe 1.5L64$0.3 \times 15$ whose frequency spectrum lies between $1-2 \mathrm{MHz}(\mathrm{B})$ being the results from the probe $\mathrm{P} 4-2 \mathrm{v}$ whose frequency spectrum lies between 2-4 MHz) for received energies as a function of skin thickness for experiment and analytical results.

TABLE I: Comparisons between the selected frequencies of experimental and analytical image

\begin{tabular}{|c|c|c|c|c|c|}
\hline $\begin{array}{c}\text { Frequency } \\
(\mathrm{MHz})\end{array}$ & $\begin{array}{c}\text { Correlation } \\
\text { coefficient }\end{array}$ & $\begin{array}{c}\text { Average shift of maximums } \\
(\mathrm{mm})\end{array}$ & $\begin{array}{c}\text { Standard deviation } \\
\text { of the shift of maximums }\end{array}$ & $\begin{array}{c}\text { Average FWHM } \\
\text { (experimental) }\end{array}$ & $\begin{array}{c}\text { Average FWHM } \\
(\text { analytical })\end{array}$ \\
\hline 1.00 & .72 & .07 & .04 & .21 & .15 \\
\hline 1.06 & .85 & .05 & .03 & .30 & .18 \\
\hline 1.13 & .86 & .05 & .04 & .15 & .19 \\
\hline 1.19 & .67 & .10 & .04 & .20 & .15 \\
\hline 2.06 & .38 & .04 & .02 & .19 & .15 \\
\hline 2.13 & .48 & .06 & .06 & .19 & .12 \\
\hline 2.19 & .55 & .09 & .05 & .20 & .11 \\
\hline 2.26 & .57 & .06 & & & .11 \\
\hline
\end{tabular}

conversion and effects due to the heterogeneous internal structure of the temporal bone, these effects should have a uniform effect across the range of skin thicknesses and frequencies and will happen regardless of the chosen frequency. The results obtained shows that US transmission in the frequency range of interest through the human skull is affected by the acoustic impedance mismatch and that the transmitted energy varies as a function of the frequency, skin thickness and bone thickness. Transmitted energy for a typical combination of skin thickness and frequency may then lead to higher SNR.

The results show that the maximum received energy level is on the order of $10^{-4}$ times the input energy. With the current configuration it is not possible to transmit more than $20 \%$ of the incident energy. The reason is that incident pressure is inversely related to the square of acoustic impedance. So if the ultrasonic wave starts from the piezoelectric domain and ends inside the brain incident pressure will be $\sqrt{\left(Z_{\text {brain }} / Z_{p}\right)}$ where $Z_{\text {brain }}$ and $Z_{p}$ are the acoustic impedances in the brain and the piezoelectric element respectively. That means:

$$
p_{\text {brain }}=\sqrt{(1.50 / 34.20)} p_{\text {incident }},
$$

where $p_{\text {incident }}$ was arbitrarily set to $1 \mathrm{~Pa}, Z_{\text {brain }}$ is 1.50 MRayl and $Z_{p}$ is 34.20 MRayl [25], which gives a maximum possible transmitted pressure of $0.20 \mathrm{~Pa}$ that in turn makes the maximum received pressure to be $0.04 \mathrm{~Pa}$ without including the attenuation effects. When including a linear attenuation factor for the bone, the received pressure level drops to $10^{-4}$ $\mathrm{Pa}$.

The interesting features in the experimental map of Fig. 7 are the three local maxima occurring for any frequency between 2-3 MHz. As explained in section II-A, a maximum transmission can occur if the thickness of the matching layer is $\lambda_{m} / 4$ and is followed by the skin and the bone thickness of odd integer multiple of $\lambda_{s} / 2, \lambda_{b} / 2$ respectively. When using $2.26 \mathrm{MHz}$ a local maximum at a skin thickness of $1.51 \mathrm{~mm}$ 

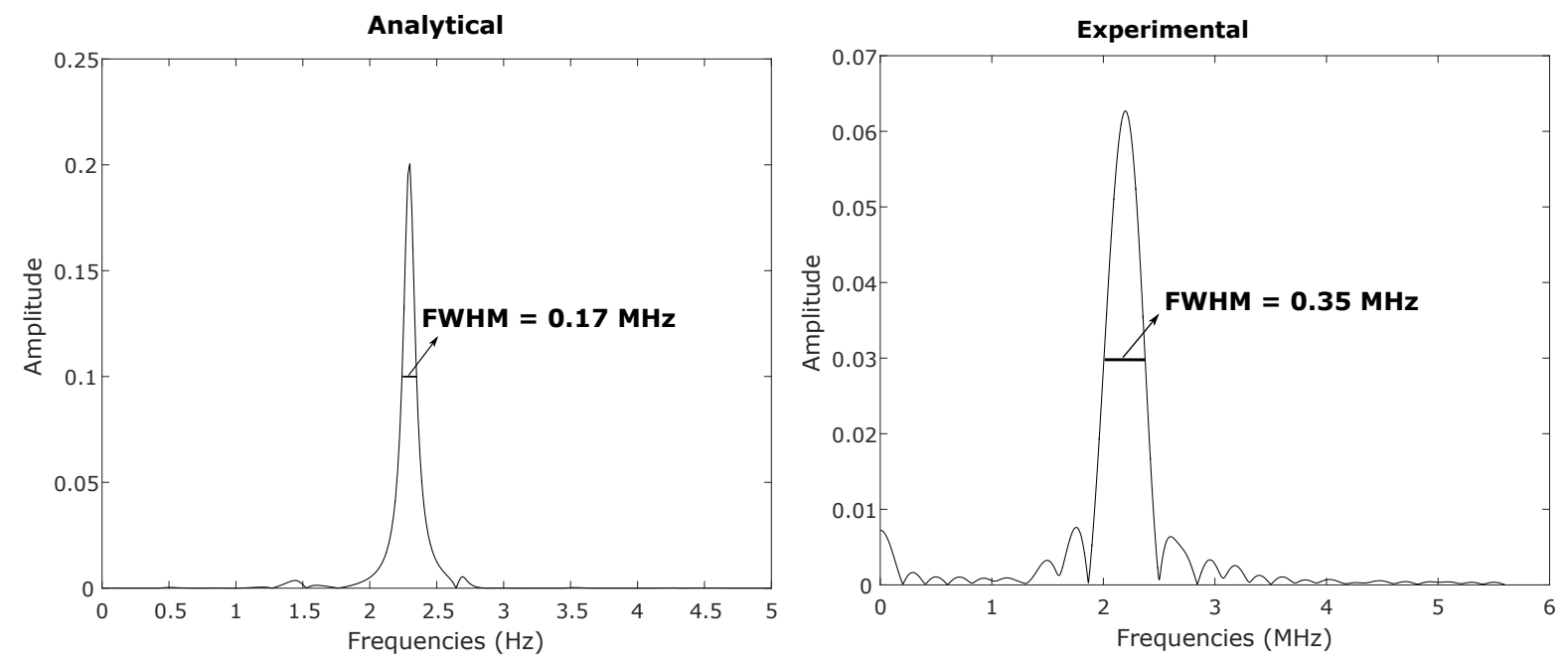

Fig. 9: Frequency spectrum for a skin thickness of $1 \mathrm{~mm}$ and a frequency of $2.20 \mathrm{MHz}$. The experimental frequency spectrum width is roughly double the analytical model spectrum. This widening of the frequency spectrum is correlated to the widening of the curves shown in Fig. 8 (B)

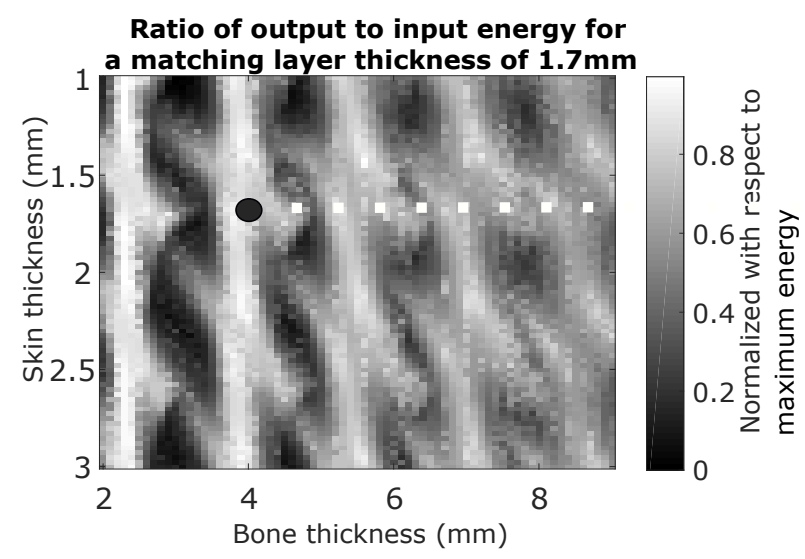

(a)

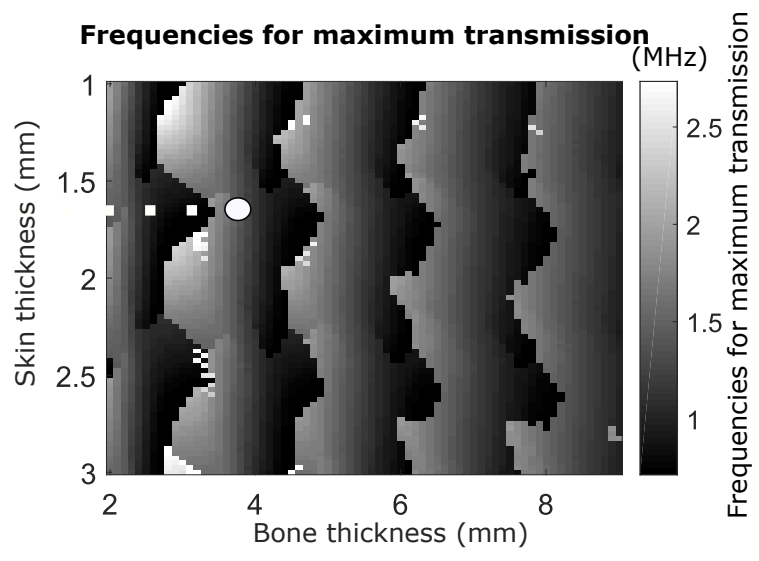

(b)

Fig. 10: $\mathrm{X}$ and Y-axes in both the figure represents skin and bone thickness respectively. A: the corresponding transmitted energy for the measured thicknesses including attenuation of $30 \mathrm{~dB} / \mathrm{cm} @ 2.25 \mathrm{MHz}$. B: variation of optimized frequencies for different skin and bone thicknesses.

can be seen. At this frequency if the matching layer thickness is $\lambda_{m} / 4$, the skin thickness becomes $\approx 5 \lambda_{s} / 2$ and the bone thickness becomes $\approx 7 \lambda_{b} / 2$ which are odd integer multiples of $\lambda / 2$ therefore lead to a local maximum energy transmission. The same concepts can be applied for all frequencies.

The average shift corresponding to the locations of the local maxima of the experimental and the analytical results fluctuated around an average of $0.06 \mathrm{~mm}$ for the selected four frequencies for both the probes with an overall standard deviation of $0.04 \mathrm{~mm}$. Thus, the model was able to accurately predict the local positions of the maxima, with small differences due to experimental errors. However, a close analysis of
Fig. 7 and Fig. 3 reveals that there is a deviation between the experimental results and the analytical results. This deviation is currently thought to be caused by the bone phantom attenuation increasing more than the predicted by assumed power law. The discrepancy between experimental and analytical results is reduced for the lower frequency probe, which supports, this interpretation. The overall correlation coefficient for the selected frequencies was 0.50 for a high frequency probe and 0.78 for a low frequency probe, showing a strong relationship between experimental and analytical results. The experimental curves are also wider than the analytical curves in the both the comparisons of Fig. 8 and this discrepancy is more pronounced 
for higher frequency probe. The reason for this widening is explained in Fig. 9, where FWHM of the experimental curve is roughly double that of the analytical curve for a selected skin thickness of $1 \mathrm{~mm}$ and for a centre frequency of 2.20 $\mathrm{MHz}$. The widening of the frequency spectrum directly leads to the widening of the curves in Fig. 8 since the ratio of the reflected to input energy is calculated based on these spectra. Physically, this means that the optimal frequency range is not as narrow as the prediction of the model. This also means there is no sudden drop of energy if the transmitted center frequency is little bit away from the optimal frequency, which is good for the practical implication of TCD.

To address the problem of using a fixed input signal for all situations, an analytical simulation was run, which gave an optimized two-dimensional map of skin and bone thicknesses for which the frequency with the highest transmission was selected as shown in Fig. 10. This figure suggests that given measurements of skin [26] and bone thickness [27], an optimal excitation frequency could be selected to optimize US transmission. The optimized two-dimensional map obtained in Fig. 10 suggests that lower frequencies from $750 \mathrm{KHz}$ to 1.7 $\mathrm{MHz}$ should be used depending on the combination of skin and bone thicknesses, which is in agreement with previous suggestions to use lower frequencies [6], [8], [9], [21], [23]. The main limitation of this study is that it does not account for inhomogeneities, anisotropy and the difference between the inner and outer structure of the skull. One more limitation of this study is that the bone phantom was considered as a single layer rather than a multilayer system. This assumption was made since an average speed of sound for the whole phantom was provided by the manufacturer and not for the individual layers. Separating the bone into multiple layers would help modeling a more realistic overall impedance mismatch but was not feasible in this study. However, the experiment remains a valid proof of concept in a relatively simple scenario to isolate the impedance mismatch effect. Apart from the skin thickness, there will be a measurable thickness of connective tissue. The thickness of connective tissue will also play a role in determining the optimal frequency selection for the measurement, but in this study the connective tissue was neglected since the acoustic impedance of subcutaneous tissues is around 1.35 MRayl [15], which is similar to soft tissue/brain so that they would be nearly transparent to ultrasound waves, and would not affect the propagation path in an adverse manner. However, one might make this model more complex by introducing this thickness. Also, this study considers only normal incidence and the effect of acoustic impedance mismatch according to the angle of entry still requires further study. Exploring these options will further enhance the knowledge related to US transmission in the skull. These effects may change the optimal selection of frequency derived by the method. Therefore, evaluating the effectiveness of frequency optimization as suggested here would require more detailed studies and experiments.

Future work will primarily focus on validating the proposed choice of centre frequency based on acoustic impedance mismatch for Doppler measurements.

\section{CONCLUSION}

TCD is a method that is used to measure blood velocity within the arteries located inside the brain but suffers from poor image quality due to less energy penetrating through the skull. The variation of US transmission as a function of frequency was considered in this paper and was due to (1) acoustic impedance mismatch and (2) attenuation. Although there are other important factors like phase aberration, scattering and diffraction inside the skull that affect transmission of ultrasound, these will affect all frequencies evenly. However by adapting the frequency to enable strong impedance matching a significant gain in transmitted energy can be achieved. In this paper, a study was conducted that allows to understand how ultrasound transmission is affected by the skin and bone thickness acoustic impedance mismatch effects. The study was performed by designing an analytical model which simulates US wave propagation from the piezoelectric element into the brain. The analytical model was validated using an experimental setup which comprised a bone phantom plate immersed in water, mimicking the acoustic properties of the skull. Comparison between analytical and experimental results, showed that the analytical model was able to accurately predict the occurrence of maximum energy transmission as a function of the frequency and the skin thickness. The average skin thickness difference between occurrence of maximum amplitudes was $0.06 \pm 0.04 \mathrm{~mm}$. Overall correlation coefficients of 0.50 and 0.78 showed a strong similarity between analytical and experimental results. More analysis indicated that an optimized frequency can be chosen depending on the measured bone and skin thicknesses. This provides an opportunity to break the barrier of ultrasound transmission through the skull.

\section{ACKNOWLEDGMENT}

This project was supported by the International Research Project (IRP) "Laboratoire Franco-Canadien de Recherches Ultrasonores" (LAFCUS) and by NSERC CREATE grant 414043-2012.

\section{REFERENCES}

[1] Smith WS, Roberts HC, Chuang NA, Ong KC, Lee TJ, Johnston SC, Dillon WP, "Safety and feasibility of a CT protocol for acute stroke: combined CT, CT angiography, and CT perfusion imaging in 53 consecutive patients", AJNR Am J Neuroradiol 2003, vol. 24, pp. 688-690

[2] R. Aaslid, "The Doppler principle applied to measurement of blood flow velocity in cerebral arteries," in Transcranial Doppler Sonography, R. A. Vienna, Ed., pp. 22-38, Springer, New York, NY, USA, 1986

[3] Thomas Devlin, MD, PhD, "A Novel Device for Pre-CT Assessment of LVOs: Initial Results from the EXPEDITE Study", SVIN, 8-11 November 2017

[4] Fry FJ, Barger JE, "Acoustical properties of human skull", J Acoust Soc Am 1978;63:pp. 1576-90.

[5] Yagita Y, Etani H, Handa N, Itoh T, Imuta N, Okamoto M, Matsumoto M, Kinoshita N, Nukada T.,"Effect of transcranial doppler intensity on successful recording in Japanese patients", Ultrasound Med Biol 1996;22:701-705.

[6] Klotzsch C, Popescu O, Berlit P, "A new 1-MHz probe for transcranial Doppler sonography in patients with inadequate temporal bone windows", Ultrasound Med Biol, vol. 24, pp. 101-103, 1998. 
[7] P. Board, M. MacKay-Lyons, P. Cunningham, M. Tse-Perron, D. Campbell, B. Fraser,S. Locke, N. MacDonald, L. Freeman, O.S. Plan, F. Statements, "Clinical Guidelines.",Heart, 2011.

[8] D. N. White, "The early development of neurosonology: I. Echoencephalography in adults," Ultrasound Med. Biol., vol. 18, pp. 115-165, 1992.

[9] J. Kollar, G. Schulte-Altedorneburg, J. Sikula, B. Fulesdi, E. B. Ringelstein, V. Mehta, L. Csiba, and D. W. Droste, "Image quality of the temporal bone window examined by transcranial doppler sonography and correlation with postmortem computed tomography measurements," Cerebrovasc. Disese, vol. 17, pp. 61-65, 2004.

[10] White, P J et al. "Local frequency dependence in transcranial ultrasound transmission" Physics in medicine and biology vol. 51,9 (2006): 2293305 "

[11] Aarnio J, Clement GT, Hynynen K. "A new ultrasound method for determining the acoustic phase shifts caused by the skull bone" Ultrasound Med Biol. 2005;31(6):771-780"

[12] Clement, G.T. et al. ,"Correlation of ultrasound phase with physical skull properties", Ultrasound in Medicine and Biology, Volume 28 , Issue 5, 617 - 624

[13] Mansour, Omar and Poepping, Tamie L and Lacefield, James C,"A beamforming method for plane wave Doppler imaging of high flow velocities", Medical Imaging 2016: Ultrasonic Imaging and Tomography, vol.-9790, 97900V, 2016

[14] M. Couade, "The advent of ultrafast ultrasound in vascular imaging: a review", J Vasc Diagn Intervent, vol.-4, pp. 9-22, 2016

[15] Shankar, Hariharan, and Paul S. Pagel. "Potential Adverse UltrasoundRelated Biological Effects: A Critical Review." Anesthesiology, vol. 115, no. 5, Nov. 2011, pp. 1109-24.

[16] Khalitov, R. Sh, S. N. Gurbatov, and I. Yu Demin, "The use of the Verasonics ultrasound system to measure shear wave velocities in CIRS phantoms", Physics of Wave Phenomena, vol. -24.1, pp. 73-76, 2016

[17] G. Pellacani and S. Seidenari, "Variations in Facial Skin Thickness and Echogenicity with Site and Age", Acta Derm Venereol, vol. 79, pp. 366399, 1999

[18] Goss SA, Johnston RL, Dunn F.,'Comprehensive compilation of empirical ultrasonic properties of mammalian tissues", J Acoust Soc Am. 1978 Aug;64(2):423-57. doi: 10.1121/1.382016. PMID

[19] H.A.M. Mahinda , O.P. Murty, "Variability in thickness of human skull bones and sternum - an autopsy experience", Journal of Forensic Medicine and Toxicology Vol. 26 No. 2, July-December 2009.

[20] Q. Yue, D. Liu, W. Wang, W. Di, D. Lin, X. Wang, and H. Luo, "Fabrication of a PMN-PT Single Crystal-Based Transcranial Doppler Transducer and the Power Regulation of Its Detection System," sensors, vol. 14, pp. 24462-71, 2014

[21] Lindsey BD, Light ED, Nicoletto HA, Bennett ER, Laskowitz DT, Smith SW, "The ultrasound brain helmet: new transducers and volume registration for in vivo simultaneous multi-transducer 3-D transcranial imaging", IEEE Trans Ultrason Ferroelectr Freq Control, vol. 58, pp.1189-1202, 2011

[22] Lindsey BD, Light ED, Nicoletto HA, Bennett ER, Laskowitz DT, Smith SW, " Simultaneous bilateral real-time 3-d transcranial ultrasound imaging at $1 \mathrm{mhz}$ through poor acoustic windows", Ultrasound in Medicine and Biology, vol. 39, pp. 721-734, 2013

[23] A. Yousefi, D. E. Goertz, and K. Hynynen, "Transcranial ShearMode Ultrasound: Assessment of Imaging Performance and Excitation Techniques", IEEE transaction on Medical Imaging, vol. 28, 2009

[24] A. Wydra and R. G. Maev, "A novel composite material specifically developed for ultrasound bone phantoms: Cortical, trabecular and skull," Phys. Med. Biol., vol. 58, pp. N303-N319, Nov. 2013

[25] J. David N. Cheeke, "Fundamentals and Application of ultrasonic waves", CRC series in pure and applied physics, 2002, ISBN 0-84930130-0

[26] R. Hesselstrand, A. Scheja, M. Wildt and A. Akesson,'High-frequency ultrasound of skin involvement in systemic sclerosis reflects oedema, extension and severity in early disease", Rheumatology 2008;47:84-87

[27] S.H. Tretbar, P.K. Plinkert, P.A. Federspil, "Accuracy of ultrasound measurements for skull bone thickness using coded signals", IEEE Transactions on Biomedical Engineering, 56 (3) (2009), pp. 733-739

[28] Hill R, El-Dardiry SMA,"A theory for optimization in the use of acoustic emission transducers", J Acoust Soc Am, vol. 67, pp. 67382,1980

[29] H. White, B. Venkatesh, "Applications of transcranial Doppler in the ICU: a review", Intensive Care Med, pp. 981-994, 2006

[30] S. W. Flax and M. o'donnell, "Phase-aberration correction using signals from point reflectors and diffuse scatterers- Basic principles,", IEEE Trans. Ultrason. Ferroelectr. Freq. Control, vol. 35, no. 6, pp. 758-767, 1988.

[31] D.L. liu and R. C. Waag, "Time-shift compensation of ultrasonic pulse focus degradation using least-mean-square error estimates of arrival time," J. Acoust. Soc. Am., vol. 95, pp. 542-555, Jan. 1994.

[32] R. Gauss, G. E. Trahey, and M. S. Soo, "Wavefront estimation in the human breast," in Proc. SPIE, 2001, pp. 172-181

[33] M. Fink, "Time reversal of ultrasonic felds. I. Basic principles," IEEE Trans. Ultrason. Ferroelectr. Freq. Control, vol. 39, no. 5, pp. 555-566, 1992.

[34] M. Pernot, G. Montaldo, M. Tanter, and M. Fink, "Ultrasonic stars" for time-reversal focusing using induced cavitation bubbles," Appl. Phys. Lett., vol. 88, no. 3, pp. 1-3, 2006.

[35] K. J. Haworth, J. B. Fowlkes, P. L. Larson, and O. D. Kripfgans, "Towards aberration correction of transcranial ultrasound using acoustic droplet vaporization," Ultrasound Med. Biol., vol. 34, pp. 435-445, Mar. 2008.

[36] J. Gateau, 1. Marsac, M. Pernot, J. F. Aubry, M. Tanter, and M. Fink, "Transcranial ultrasonic therapy based on time reversal of acoustically induced cavitation bubble signature," IEEE Trans. Biomed. Eng., vol. 57, pp. 134-144, Jan. 2009 